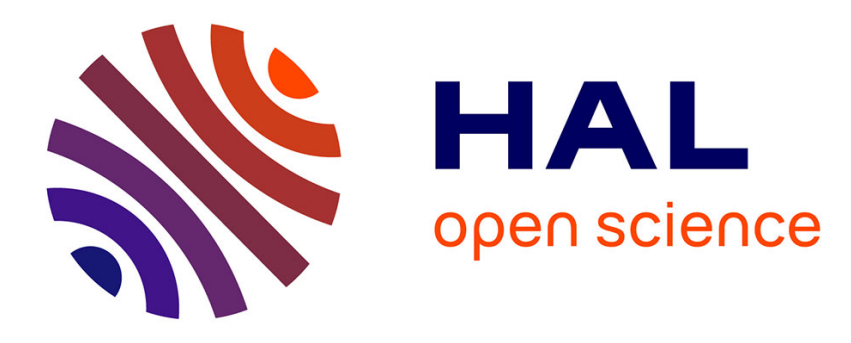

\title{
Constellation design with deep learning for downlink non-orthogonal multiple access
}

Florence Alberge

\section{To cite this version:}

Florence Alberge. Constellation design with deep learning for downlink non-orthogonal multiple access. IEEE International Symposium on Personal, Indoor and Mobile Radio Communications (PIMRC 2018), Sep 2018, Bologna, Italy. hal-01894501

\section{HAL Id: hal-01894501 \\ https://hal-centralesupelec.archives-ouvertes.fr/hal-01894501}

Submitted on 28 Feb 2020

HAL is a multi-disciplinary open access archive for the deposit and dissemination of scientific research documents, whether they are published or not. The documents may come from teaching and research institutions in France or abroad, or from public or private research centers.
L'archive ouverte pluridisciplinaire HAL, est destinée au dépôt et à la diffusion de documents scientifiques de niveau recherche, publiés ou non, émanant des établissements d'enseignement et de recherche français ou étrangers, des laboratoires publics ou privés. 


\title{
Constellation design with deep learning for downlink non-orthogonal multiple access
}

\author{
F. Alberge \\ L2S, Univ. Paris-Sud - CNRS - CentraleSupélec, Université Paris-Saclay, F-91192, Gif-sur-Yvette, France. \\ Tel: +33169851757 - E-mail:florence.alberge@12s.centralesupelec.fr
}

\begin{abstract}
The non-orthogonal multiple access (NOMA) technique is considered as a key component for the next generation cellular system. In downlink NOMA, the constellation of several users are superposed for transmission. The resulting super-constellation needs to be carefully designed for allowing recovering of the data at the receiver side. A deep learning method for constellation optimization is proposed here in the context of downlink NOMA communications. The method is based on an analogy between auto-encoders, a powerful tool in neural networks, and communication systems. Simulation results have verified the effectiveness of this method for both constellation design and optimization of the individual receivers of the users. The optimized encoder/decoder can be successfully combined with iterative error-correction devices such as turbo-codes or LDPC and can be integrated in current communication systems. This technique is quite general and can be used for point-to-point communication as well as for multi-user access under various channel conditions.
\end{abstract}

\section{INTRODUCTION}

Non-orthogonal multiple access (NOMA) has received significant attention for the fifth generation $(5 \mathrm{G})$ wireless networks. NOMA is expected to meet the increasing need of data transmission rate and demands on massive connectivity and high reliability [1], [2], [3]. The key idea behind NOMA is to serve multiple users in the same resource block by exploiting the channel gain differences. Non-orthogonal techniques have the potential to achieve the whole capacity region for both downlink and uplink channels [4]. In downlink NOMA, signals intended for different users are superposed at the base station before transmission through the same resource. In general, successive interference cancellation (SIC) is implemented at the receiver. The strong user estimates and cancels the signals of the weak users before decoding its own message. The weak user treats the signal of the other users as interference or noise enabling a direct decoding of its own message. Therefore, super-constellation design is a crucial point for enhanced performance with NOMA. This question is related to constellation design for the 2-user broadcast channel for which theoretical results can be found in [5], [6]. In practical systems however, constellations with finite cardinality are employed such as QAM or PAM constellations. Recently, several publications have considered optimal super-constellation design based on off-the-shelf constellations. The superposition of BPSK signals with equal power allocation factor (PAF) is investigated in [7] whereas the superposition of BPSK signals with unequal PAF is studied in [8]. In [9], superposition coding with QPSK signals is proposed including power allocation policy and phase-shift. Other contributions focused on the angles of rotation of the individual constellations [10] in order to optimize the spatial diversity. Higher order modulation schemes are considered in [11] where analytical expressions are obtained for the Bit error rate (BER) in NOMA with $M_{1}$-QAM (or BPSK) $+M_{2}$ QAM with unequal PAF. In [11], neither the labeling nor possible angles of rotation are studied whereas the allocated power and phase rotation are optimized in [12]. In [13], two-user superposition transmission scheme with QAM input is studied. An exhaustive search for the optimal PAF based on mutual information is proposed which is computationally prohibited. Another amount of research focus on coded data, labeling and receiver design. It is proved in [14] that Gray-mapping NOMA should be preferred to non-Gray mapping when maximum likelihood (ML) detection is implemented at the receiver. A simple scheme based on PAM and turbocodes is studied in [15] to realize the promised gains of downlink NOMA. Finding good super-constellations require to choose in an optimal manner the individual constellations, their respective power and angles of rotation, labeling and a proper receiver for each user. In most of the previous publications these requirements where addressed separately. The main contribution of this paper is to provide a joint optimization framework for super-constellation/receiver design. Our approach does not require to perform SIC at the receiver and is therefore not subject to error propagation in contrast to [12]. Deep learning (DL) is a branch of machine learning research which has been introduced to allow machines and computer systems to improve with experience and data accumulation. Recent years have seen renewed interest for the study of DL. Auto-encoders (AEs) play a fundamental role in machine learning. They were first introduced in [16] to address the problem of unsupervised learning. An AE is a neural network that is trained 
to copy its input at its output [17]. Internally, an AE consists of two parts usually termed as encoder and decoder. The encoder is trained to produce a representation of the input with desired properties (compression, robustness to harsh environment,etc.) whereas the decoder consists in recovering the input from this representation. Neural network have been used recently in several publications from the communication community. For example, a methodology is proposed in [18] for learning symbol basis functions from existing modulation signals. More recently an algorithm for signal detection for molecular communication has been proposed in [19] demonstrating the ability of neural networks to detect symbols even when the channel is unknown and nonlinear.

We do believe that AEs are the right tool for constellation design in NOMA. Indeed, the encoder can be trained to optimize the constellation whereas the decoders are trained to recover the binary message of each user from the received data. The strengths of the proposed methodology are listed below. The criterion to be optimized is a lower bound of the mutual information which is usually considered in constellation design. Neither an analytical expression of the mutual information nor an a priori on the geometry of the constellation are needed for the optimization process which is in contrast with previous works. The role of the encoder is to map a binary word to a specific constellation point meaning that the constellation point positions and the labeling are optimized jointly. The proposed mapping/de-mapping solution can be used in combination with powerful channel decoders such that turbo-codes or Low density Parity-Check codes (LDPC) and is fully compatible with wireless communications schemes already deployed. The validity of the proposed scheme will be emphasized in the simulation part.

\section{SYSTEM DESCRIPTION}

\section{A. Auto-encoders}

An AE is a neural network that is trained for learning a useful representation $Y$ of an input $X$. The simplest form of an $\mathrm{AE}$ is a feedforward, non-recurrent neural network organized in two different blocks usually termed as encoder and decoder. Let $\mathbf{x}$ denote a given input vector with size $d$ and $\mathbf{y}$ the resulting output vector with size $d^{\prime}$ then a one-layer encoder is given by the deterministic mapping

$$
\mathbf{y}=f_{\theta}^{d \rightarrow d^{\prime}, s}(\mathbf{x})=s(\mathbf{W} \mathbf{x}+\mathbf{b})
$$

where $\theta=\{\mathbf{W}, \mathbf{b}\}$ is the set of parameters to be optimized and the respective size of $\mathbf{W}$ and $\mathbf{b}$ are $d^{\prime} \times d$ and $d^{\prime} \times 1$. The function $s($.$) is either a non-linear$ function or could also be the identity mapping. This is the simplest configuration however, in general, an $\mathrm{AE}$ is the concatenation of several layers for which the output of the previous layer is used as an input for the current layer. The task of the decoder is to recover the input $\mathbf{x}$ from the hidden representation $\mathbf{y}$ through

$$
\mathbf{z}=f_{\theta^{\prime}}^{d^{\prime} \rightarrow d, s}(\mathbf{x})=s\left(\mathbf{W}^{\prime} \mathbf{y}+\mathbf{b}^{\prime}\right)
$$

where $\theta^{\prime}=\left\{\mathbf{W}^{\prime}, \mathbf{b}^{\prime}\right\}$ and where $s($.$) is not necessarily$ the same function than in (1). The network is then trained in order to optimize the reconstruction error $L(\mathbf{x}, \mathbf{z})=-\log p(\mathbf{x} \mid \mathbf{z})$. Here, binary input will be considered and our problem can be seen as a binary classification problem with the particularity that the data $\mathbf{x}$ are also the target labels. With binary classification, the adequate reconstruction error is the cross-entropy loss [20], namely

$$
L(\mathbf{x}, \mathbf{z})=-\sum_{j}\left(\mathbf{x}_{j} \log \mathbf{z}_{j}+\left(1-\mathbf{x}_{j}\right) \log \left(1-\mathbf{z}_{j}\right)\right)
$$

In that case, $X_{j} \mid \mathbf{z} \sim \mathcal{B}\left(\mathbf{z}_{j}\right)$ where $\mathcal{B}$ stands for the Bernouilli and $\mathbf{z}_{j}$ is such that $\mathbf{z}_{j}=P\left(X_{j}=1 \mid \mathbf{z}\right)$. The training of the network is performed by solving the following optimization problem

$$
\arg \min _{\theta, \theta^{\prime}} \mathbb{E}_{X}[L(X, Z)]
$$

When the distribution of the input is unknown, the expectation is replaced by the empirical average over the training samples. It is proven in [20] that the optimization problem in (4) is equivalent to the maximization of a lower bound on the mutual information $I(X ; Y)$.

\section{B. Downlink NOMA}

In downlink NOMA, under consideration here, a super-constellation is transmitted that comes from the superposition of the signals of the individual users. In the 2 -user case, the transmitted signal reads

$$
\mathbf{y}=\sqrt{p_{1}} \mathbf{y}_{1}+\sqrt{p_{2}} \mathbf{y}_{2}
$$

where $p_{1}$ and $p_{2}$ are respectively the power of the first and second user and $\mathbf{y}_{1}$ and $\mathbf{y}_{2}$ are the corresponding signals of each user. The super-symbol is normalized such that $E\left[|\mathbf{y}|^{2}\right]=1$ and transmitted to the users

$$
\mathbf{r}_{i}=\mathbf{h}_{i} \mathbf{y}+\mathbf{n}_{i}
$$

where $\mathbf{h}_{i}$ is the complex channel coefficient between the base station and user $i, \mathbf{n}_{i}$ is the AWGN noise with spectral power density $N_{0, i}$ and $S N R_{i}$ is the signal to noise ratio (in $\mathrm{dB}$ ) of user $i$. We will consider here both AWGN and Rayleigh block-fading channels. The AWGN channel is the appropriate model for downlink NOMA with OFDM [21]. Without loss of generality, the near user is user 1 whereas user 2 is the far user. By assigning a sufficient power to user 2 the interference is expected to be sufficiently small to enable user 2 to recover its useful information. 


\section{AN AUTO-ENCODER FOR DOWNLINK NOMA}

\section{A. General description}

For sake of simplicity, the proposed structure is described in the two users case. Generalization to a higher number of users is straightforward. Let $\mathbf{x}=\left[\mathbf{x}_{\mathbf{1}} ; \mathbf{x}_{\mathbf{2}}\right]$ denote the input of the AE. At the same time, $\mathbf{x}$ is the binary message which is composed of $k_{1}$ bits at destination of user 1 forming $\mathbf{x}_{1}$, and $k_{2}$ bits at destination of user 2 forming $\mathbf{x}_{2}$. The total number of bits is $k=k_{1}+k_{2}$. Therefore, the super-constellation with size $M=2^{k}$ is the superposition of two constellations with size $2^{k_{1}}$ and $2^{k_{2}}$. The output of the encoder is $\mathbf{y}$ (transmitted symbol and constellation point). This is a vector of size 2 containing the real and imaginary parts of the transmitted symbol. In AEs, noise is usually added at the input of the $\mathrm{AE}$ to enhance the generalization capability of the network or to implement a denoising auto-encoder. Here, the noise is added at the output of the encoder in order to simulate the transmission channel in (6) between the base station and the users. The task of receiver 2 is to provide an accurate estimation of $\mathbf{x}_{2}$ based on the received symbol $\mathbf{r}_{2}$ whereas receiver 1 is trained to recover both $\mathbf{x}_{1}$ and $\mathbf{x}_{2}$ like in SIC where the near user should be able to decode the message of the far user. Note however that SIC is not implemented here. Instead the network is trained for learning the task which consists in recovering the emitted message. In the evaluation phase, the BER of user 1 is computed based on the sole $k_{1}$ bits in $\mathbf{x}_{1}$ even if the network has also been trained to recover $\mathbf{x}_{2}$. The loss function is the sum of two contributions

$$
L_{r_{1}}(\mathbf{x}, \mathbf{z})+L_{r_{2}}\left(\mathbf{x}_{2}, \mathbf{z}_{2}\right)
$$

where $L$ is defined in (3) and where $r_{i}$ is a reference to the channel in which the data are transmitted.

\section{B. Network configuration}

One of the most intricate task in deep learning is to design the network (number of layers, number of hidden units/layer, number of epochs, choice of the optimizer, batch size, etc.) since there are no general guidelines for that. The proposed structure is depicted in Fig. 1 and is more precisely described below:

- Encoder. The bit to symbol mapping can be carried out by a linear operation. The encoder is therefore a single layer with input length $k$ and output length 2. Then, $\mathbf{y}$ reads

$$
\mathbf{y}=f_{\theta_{1}}^{k \rightarrow 2, l}(\mathbf{x})=\mathbf{W}_{1} \mathbf{x}+\mathbf{b}_{1}
$$

where $l$ means linear activation function.

- Decoder. Following [22], decoder1 and decoder2 are constituted of 3 layers with respective output length $[128,64,32]$ followed by an output layer of output length $k$ for decoder 1 and $k_{2}$ for decoder 2 .

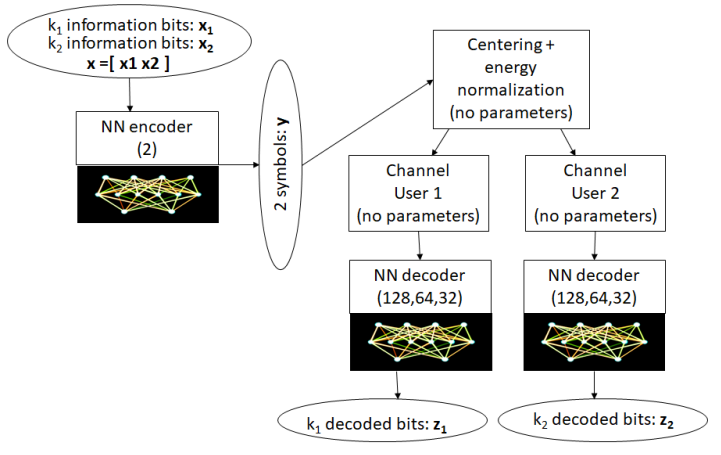

Figure 1. Deep learning structure for 2-user mapping

Formally, $\mathbf{z}$ and $\mathbf{z}_{2}$ which are soft estimates of $\mathbf{x}$ and $\mathbf{x}_{2}$ respectively are given by

$\mathbf{z}=f_{\theta_{1}^{\prime}}^{2 \rightarrow 128, r} \circ f_{\theta_{2}^{\prime}}^{128 \rightarrow 64, r} \circ f_{\theta_{3}^{\prime}}^{64 \rightarrow 32, r} \circ f_{\theta_{4}^{\prime}}^{32 \rightarrow k, s}\left(\mathbf{r}_{\mathbf{1}}\right)$
$\mathbf{z}_{\mathbf{2}}=f_{\theta_{5}^{\prime}}^{2 \rightarrow 128, r} \circ f_{\theta_{6}^{\prime}}^{128 \rightarrow 64, r} \circ f_{\theta_{7}^{\prime}}^{64 \rightarrow 32, r} \circ f_{\theta_{8}^{\prime}}^{32 \rightarrow k_{2}, s}\left(\mathbf{r}_{\mathbf{2}}\right)$

where $r$ and $s$ stand respectively for the rectified linear unit (relu) and sigmoid activation functions.

- Optimization. The stochastic gradient descent (SGD) with learning rate $\lambda=0.1$ is used in the optimization process. Based on our experiments, the number of epochs is fixed to $2^{19}$ and the batchsize is $2^{k}$ which corresponds to the whole set of input data. The gradient of the loss function is calculated over the entire input set during each epoch as a consequence dropout is useless here.

In our architecture, the channel layer generates a new realization of the noise and of the channel coefficient each time it is used. The optimization problem is solved with a unit-norm constraint on the columns of matrices $\mathbf{W}$ to avoid scaling effects. The goal of training is to find the optimal set of parameters. The optimized mapping and demapping blocks are given by (8) and (9-10) when the parameters are set to the optimal ones. The training of the neural network is implemented with Keras which is a convenient high-level abstraction frontend for Theano or Tensorflow. This structure can be generalized to any number of users.

\section{Simulation Results}

\section{A. Case 1: uncoded data, 2 users}

Analytical expressions are given in [11] for the estimation of each NOMA's user bit probability for the 2 users case where the superconstellation is the superposition of M-QAM and/or BPSK constellations and the channel is AWGN. Let denote $B E R_{i}$ the BER of user $i$, it is thus possible to compute, for a given SNR value, the theoretical BER such that $B E R_{1}=B E R_{2}$. These results are used as a benchmark. On the other side, the $\mathrm{AE}$ is trained with the loss in (3) in which the individual 


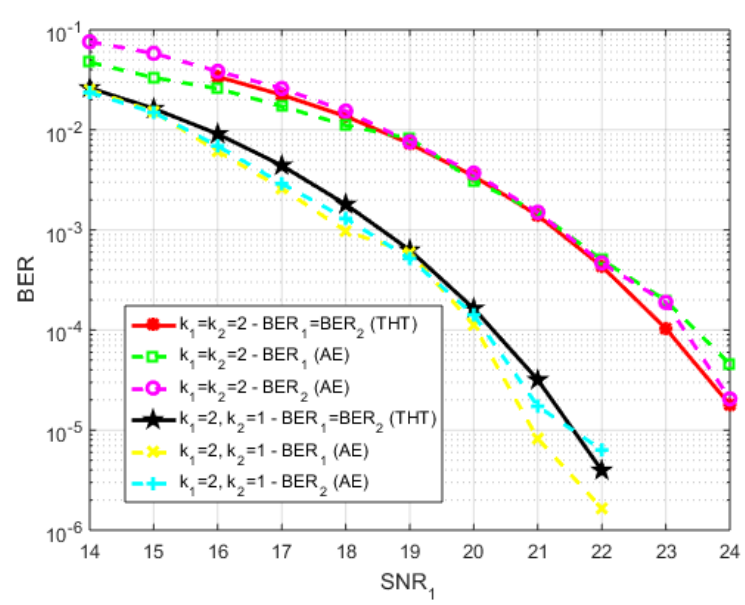

Figure 2. BER vs $S N R_{1}(\Delta S N R=9 d B)-$ THT $=$ theoretical [11] - $\mathrm{AE}=$ superconstellations optimized with the $\mathrm{AE}$.

losses are given the same weight. The users are such that $\triangle S N R=S N R_{1}-S N R_{2}=9 d B$. We consider the two following situations: $\left(k_{1}=2, k_{2}=2\right)$ and $\left(k_{1}=2, k_{2}=1\right)$. For computing the theoretical BER, it is assumed that the constellation is QPSK+QPSK when $\left(k_{1}=2, k_{2}=2\right)$ and QPSK+BPSK when $\left(k_{1}=2, k_{2}=1\right)$. The numerical results are given in Fig. 2 where BER (THT) is the theoretical target. We can first observe that, $B E R_{1} \approx B E R_{2}$ which is what was expected by choosing the same weights for the losses of the individual users. The second observation is that, regardless of the value of the SNR, $B E R_{1}$ and $B E R_{2}$ are very close to the theoretical BER hence the $\mathrm{AE}$ always yields a valid solution. For $\left(k_{1}=2, k_{2}=2\right)$, the super-constellation obtained with the AE are rotated QPSK+QPSK whereas for $\left(k_{1}=2, k_{2}=1\right)$ the superconstellation is in general a 4PAM+BPSK this is the reason why both $B E R_{1}$ and $B E R_{2}$ can be slightly better than the theoretical BER. One of the advantage of deep learning in this scenario is that the labeling, the positions of the constellation points as well as the decoder are jointly optimized and does not require any prior information, knowledge or intuition.

\section{B. Case 2: uncoded data, 3 users}

The 3 users case is now considered. The loss is the sum of three contributions namely

$$
L_{r_{1}}(\mathbf{x}, \mathbf{z})+L_{\mathbf{r}_{2}}\left(\left[\mathbf{x}_{\mathbf{2}}, \mathbf{x}_{\mathbf{3}}\right],\left[\mathbf{z}_{2}, \mathbf{z}_{3}\right]\right)+L_{\mathbf{r}_{3}}\left(\mathbf{x}_{\mathbf{3}}, \mathbf{z}_{3}\right)
$$

where the strongest users are trained to decode their own message as well as the messages of the weaker users. The constellations $\mathcal{X}_{A E}$ are given in Fig. 3 and 4. We can observe that for all the considered settings the $\mathrm{AE}$ is able to select an appropriate geometry and PAF.
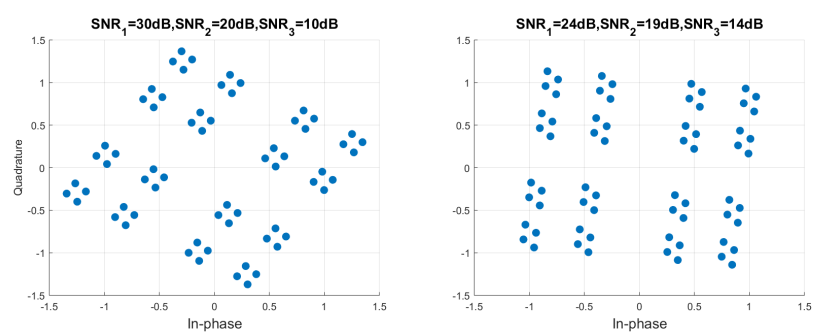

Figure 3. $\mathcal{X}_{A E}$ with $\left(k_{1}=2, k_{2}=2, k_{3}=2\right)$ and AWGN.
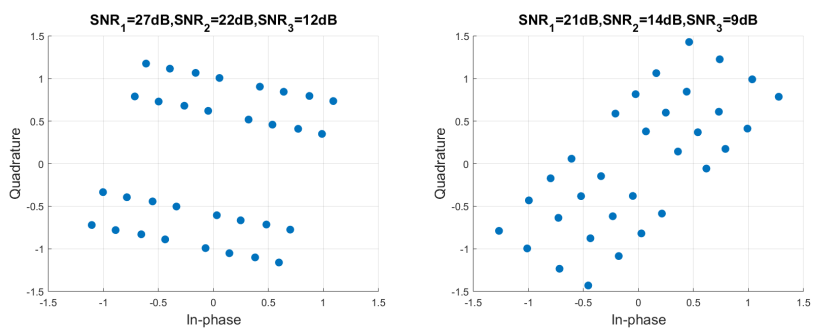

Figure 4. $\mathcal{X}_{A E}$ with $\left(k_{1}=2, k_{2}=2, k_{3}=1\right)$ and AWGN.

\section{Case 3: coded data, 2 users}

Even if the superconstellations are optimized with uncoded data, the compatibility with error-correction devices must be considered for practical use. For that purpose, the binary message is encoded with off-theshelf DVB-S2 LDPC code. We want here to serve two users with $\triangle S N R \approx 9 d B$. The superconstellation has a cardinality $|\mathcal{X}|=16$ and $\left(k_{1}=2, k_{2}=2\right)$. We first select the constellation which has the best results in terms of BER for the whole range of SNRs. The best super-constellation is obtained with $\left(S N R_{1}=11 \mathrm{~dB}\right.$, $\left.S N R_{2}=2 d B\right)$ as training SNRs for the AWGN channel and with $\left(S N R_{1}=12 d B, S N R_{2}=3 d B\right)$ as training SNRs for the Rayleigh block-fading channel with known channel state information at the receiver. They are given in Fig. 5. Except for the angle of rotation, the two constellations exhibit a very similar shape with identical PAF. Hence, like in point-to-point communications, the same constellation can be used for both AWGN and Rayleigh fading channels. The communication chain considered here is constituted of two binary messages with length $64800 R$ where $R$ is the code rate. These messages are encoded with DVBS2 LDPC. The codewords are then splitted into blocks of length $k_{1}$ and $k_{2}$ and mapped to the superconstellation in Fig. 5 and broadcasted to the users. At the receivers side, the optimized decoder computes the soft estimates $\mathbf{z}_{1}$ or $\mathbf{z}_{2}$ based on the observations $\mathbf{r}_{1}$ for user 1 and $\mathbf{r}_{2}$ for user 2. Since $\mathbf{z}_{1}$ or $\mathbf{z}_{2}$ are probabilities, the corresponding log-likelihood ratios are computed and serves as an input to the LDPC decoder. The BER are given in Fig. 6 for different values of $R$ and for the AWGN and Rayleigh block-fading channel. As can be seen, numerical results 

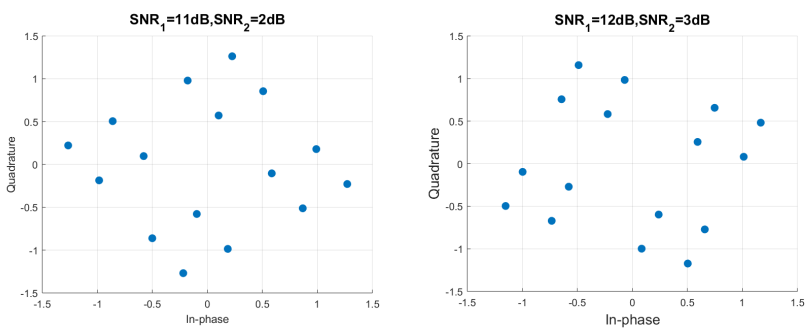

Figure 5. $\mathcal{X}_{A E}$ with $\left(k_{1}=2, k_{2}=2\right)$. Left: AWGN - Right: Rayleigh channel.

\begin{tabular}{|l|l|l|l||l|l|l|l|}
\hline Fig. & $P_{1}$ & $P_{2}$ & $P_{3}$ & Fig. & $P_{1}$ & $P_{2}$ & $P_{3}$ \\
\hline 3-L & 0.010 & 0.134 & 0.856 & $3-\mathrm{R}$ & 0.012 & 0.124 & 0.864 \\
\hline 4-L & 0.066 & 0.326 & 0.608 & 4-R & 0.155 & 0.161 & 0.684 \\
\hline 5-L & 0.123 & 0.877 & & 5-R & 0.098 & 0.902 & \\
\hline
\end{tabular}

Table 1

PAF FOR $\mathcal{X}_{A E}$ IN FIG. 3-5. L= LEFT, R= RIGHT.

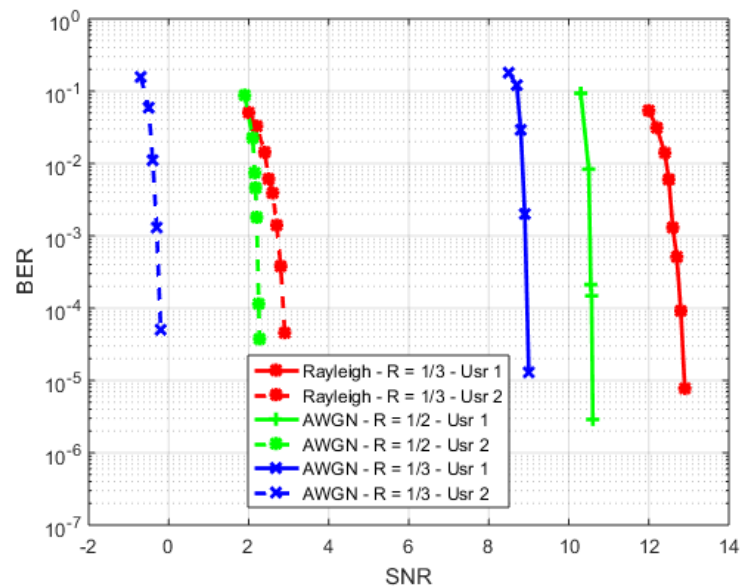

Figure 6. BER vs SNR - DVBS2-LDPC 2 users $\left(k_{1}=k_{2}=2\right)$.

demonstrates the effectiveness of the proposed method in the context of downlink NOMA. The PAF for $\mathcal{X}_{A E}$ in Figs. 3 to 5 are given in table I.

\section{CONCLUSIONS}

The efficiency of AEs for constellation optimization in downlink NOMA has been proved in this article. The optimization method is general and encompasses all possible configurations (number of users and relative position of the users). Analytical expressions of the BER or of the mutual information are not required and the method can thus be generalized to other impairments such as phase noise or other type of interferences. After the optimization process, the mapping and demapping blocks can be plugged into current communication systems. In particular the de-mapping sub-block is compatible with hard input as well as soft input devices. In this paper, off-line optimization was considered. Future research include on-line optimization as well as constellation design for secure communications.

\section{REFERENCES}

[1] Z. Ding, Z. Yang, P. Fan, and H. V. Poor, "On the Performance of Non-Orthogonal Multiple Access in 5G Systems with Randomly Deployed Users," IEEE Signal Processing Letters, vol. 21, no. 12, pp. 1501-1505, Dec 2014.

[2] L. Dai, B. Wang, Y. Yuan, S. Han, C. 1. I, and Z. Wang, "Non-orthogonal multiple access for 5G: solutions, challenges, opportunities, and future research trends," IEEE Communications Magazine, vol. 53, no. 9, pp. 74-81, September 2015.

[3] S. M. R. Islam, N. Avazov, O. A. Dobre, and K. s. Kwak, "Power-Domain Non-Orthogonal Multiple Access (NOMA) in 5G Systems: Potentials and Challenges," IEEE Communications Surveys Tutorials, vol. 19, no. 2, pp. 721-742, 2017.

[4] Y. Saito, Y. Kishiyama, A. Benjebbour, T. Nakamura, A. Li, and K. Higuchi, "Non-orthogonal multiple access (noma) for cellular future radio access," in 2013 IEEE 77th Vehicular Technology Conference (VTC Spring), June 2013, pp. 1-5.

[5] T. Cover, "Broadcast channels," IEEE Transactions on Information Theory, vol. 18, no. 1, pp. 2-14, Jan 1972.

[6] P. Bergmans, "A simple converse for broadcast channels with additive white gaussian noise (corresp.)," IEEE Transactions on Information Theory, vol. 20, no. 2, pp. 279-280, Mar 1974.

[7] R. Zhang and L. Hanzo, "A unified treatment of superposition coding aided communications: Theory and practice," IEEE Commun Surveys Tutorials, vol. 13, no. 3, pp. 503-520, Third 2011.

[8] P. A. Hoeher and T. Wo, "Superposition modulation: myths and facts," IEEE Communications Magazine, vol. 49, no. 12, pp. 110-116, December 2011.

[9] L. Ping, J. Tong, X. Yuan, and Q. Guo, "Superposition coded modulation and iterative linear mmse detection," IEEE Journal on Selected Areas in Communications, vol. 27, no. 6, pp. 9951004, August 2009.

[10] J. Zhang, X. Wang, T. Hasegawa, and T. Kubo, "Downlink NonOrthogonal Multiple Access (NOMA) Constellation Rotation," in 2016 IEEE 84th Vehicular Technology Conference (VTC-Fall), Sept 2016, pp. 1-5.

[11] E. C. Cejudo, H. Zhu, and O. Alluhaibi, "On the power allocation and constellation selection in downlink noma," in IEEE 86th Vehicular Technology Conference, Sept 2017, pp. 1-5.

[12] W. Wang and W. Zhang, "Optimal signal constellation for downlink two-user noma," in 2017 23rd Asia-Pacific Conference on Communications (APCC), Dec 2017, pp. 1-6.

[13] J. Choi, "On the power allocation for a practical multiuser superposition scheme in noma systems," IEEE Commun. Letters, vol. 20, no. 3, pp. 438-441, March 2016.

[14] H. Lee, S. Kim, and J. H. Lim, "Multiuser superposition transmission (must) for lte-a systems," in 2016 IEEE International Conference on Communications (ICC), May 2016, pp. 1-6.

[15] S. L. Shieh and Y. C. Huang, "A simple scheme for realizing the promised gains of downlink nonorthogonal multiple access," IEEE Trans. on Commun., vol. 64, no. 4, pp. 1624-1635, 2016.

[16] D. Rumelhart, G. Hinton, and R. Williams, Parallel Distributed Processing. Vol 1: Foundations. MIT Press, Cambridge, MA, 1986, ch. Learning internal representations by error propagation

[17] I. Goodfellow, Y. Bengio, and A. Courville, Deep Learning. MIT Press, 2016, http://www.deeplearningbook.org.

[18] T. J. O'Shea, J. Corgan, and T. C. Clancy, "Unsupervised representation learning of structured radio communication signals," in 2016 First International Workshop on Sensing, Processing and Learning for Intelligent Machines (SPLINE), July 2016, pp. 1-5.

[19] N. Farsad and A. J. Goldsmith, "Detection algorithms for communication systems using deep learning," CoRR, 2017. [Online]. Available: http://arxiv.org/abs/1705.08044

[20] P. Vincent and al, "Stacked denoising auto-encoders : learning useful representation in a deep network with a local denoising criterion," Journ. of Machine Learning Research, no. 11, pp. 3371-3408, 2010.

[21] S. L. Shieh and al, "On Gray Labeling for Downlink NonOrthogonal Multiple Access Without SIC," IEEE Commun. Letters, vol. 20, no. 9, pp. 1721-1724, Sept 2016.

[22] T. Gruber, S. Cammerer, J. Hoydis, and S. ten Brink, "On deep learning-based channel decoding," in CISS'17, 2017. 